\title{
Temperature Dependent Electrical and Micromechanical Properties of Lanthanum Titanate with Additions of Yttria
}

\author{
Dr. Jon C. Goldsby \\ National Aeronautics and Space Administration \\ John H. Glenn Research Center \\ 21000 Brookpark Road Mail Stop 106-5 \\ Cleveland, OH. 44135
}

Introduction

Lanthanum titanate $\left(\mathrm{La}_{2} \mathrm{Ti}_{2} \mathrm{O}_{7}\right)$ a layered distorted perovskite (1) with space group Pna2 $2_{1}$ (Fig 1) has been shown to have potential as a high temperature piezoelectric (2).

However this highly refractory oxide compound must be consolidated at relatively high temperatures $\sim 1400^{\circ} \mathrm{C}$. Commercial $\mathrm{La}_{2} \mathrm{Ti}_{2} \mathrm{O}_{7}$ powders were mechanically alloyed with additions of $\mathrm{Y}_{2} \mathrm{O}_{3}$ to lower the consolidation temperature by $300^{\circ} \mathrm{C}$ and to provide post processing mechanical stability. Temperature dependent electrical, elastic and anelastic behavior were selected as nondestructive means of evaluating the effects of yttria on the properties of this ferroceramic material.

Method and Materials

Lanthanum titanate powders were hot pressed at $1100^{\circ} \mathrm{C}$ into bars and from air sintered from aqueous based slurry tapes, for the mechanical and electrical measurements, respectively. Anelastic behavior as a function of temperature was determined by observation of the time dependent amplitude free decay of the sample. Temperature dependent elastic properties were determined by establishing continuous flexural vibrations in the material at its lowest resonance frequency at $3 \mathrm{kHz}$. Two terminal electronic characterizations were performed using a Solartron Analytical frequency response analyzer, model 1260 with the dielectric interface model 1296. Polarization measurements were performed with a Radiant Technologies Precision ferroelectric tester. Density and electron microscopy was performed on selected test samples. The partial density of states and band structure was calculated based upon energy minimization using density functional pseudo potentials. The computations were performed with Materials Studio's CASTEP atomic simulation software code. The calculations were run on a LINUX based operating system using dual Itanium 64 bit $1 \mathrm{GHz}$ processors.

Results and Discussion

The results (Fig 2) of hot pressing lanthanum titanate with increasing amounts of yttria shows an increase in density as well as elastic modulus approaching the theoretical value of $5.78 \mathrm{~g} / \mathrm{cc}$ and $181 \mathrm{GPa}$, respectively. Scanning electron micrographs (Fig 3) reveal numerous irregular and isolated pores, which are likely to prohibit full densification. However Fig.4 illustrates a typical microstructure with addition of yttria, only $0.1 \mathrm{~mol} \%$ in this case. The light and dark grey regions are various phases of lanthanum titanate along with alumina inclusions (black), which were likely introduced in the milling process. To help determine the nature of the yttria/ lanthanum titanate interface single 
crystals were grown (Dr. Ali Sayir, NASA Glenn) and high resolution transmission microscopy was performed (Dr. Y.L. Chen, NASA Glenn). The HRTEM micrograph (Fig 5) reveals the yttria/ lanthanum titanate interface with a coherent intermediate compound between them, most likely yttrium titanate.

Applying an external electric field to the samples at elevated temperatures reveals hysterisis like behavior (Fig. 6). Saturation occurs at $360^{\circ} \mathrm{C}$ and $1.4 \mathrm{kV} / \mathrm{cm}$. Temperature dependent, low electric potential $(\mathrm{E}=0.1$ volt $\mathrm{rms})$, complex impedance tests were performed on platinum/ ferroelectric / platinum cells to determine the electronic losses. Figures 7 and 8 illustrate the real and imaginary impedance of lanthanum titanate at $600^{\circ} \mathrm{C}$ in air. The imaginary part of the complex impedance (Fig. 9) plotted as a function of frequency and temperature reveals a thermally activated peak, which decreases in magnitude as the temperature increases. This is consistent with a mechanism whereby the crystal lattice expands reducing the reorientation energy of electro-elastic defects. The calculated band structure and partial density of states (Fig. 10) gives insight into the electronic mechanisms of this insulating ferroelectric and possible doping scheme to alter its electronic properties (3). A direct $2 \mathrm{eV}$ band gap is predicted in the $\mathbf{G}$ symmetry direction in the Brillouin zone. The valance band is made of hybridized titanium $\mathbf{3 d}$ and oxygen $\mathbf{2 p}$ orbitals while the conduction band consists of a $\mathbf{p}+\mathbf{d}$ hybrid orbital made largely from the lanthanum $\mathbf{5 d}$ and titanium $3 \mathbf{d}$ atomic orbitals. Smaller contributions to the valence band come from the lanthanum $5 p$ and oxygen $2 p$ levels. Hence for this $\mathrm{A}_{2} \mathrm{~B}_{2} \mathrm{O}_{7}$ compound, the $\mathrm{A}$ site is best suited to host single valence cation substitutions, while multi-valence substitutions would best occur at the $\mathrm{B}$ site.

One order of magnitude reduction in yttria content results in a reduction in elastic modulus (Fig. 11) below 5 mol\% yttria differences in both the stiffness and in the elastic temperature coefficient are not substantial, while still producing excellent mechanical properties at elevated temperatures. One mechanical loss peak (Fig 12) appears at $466^{\circ} \mathrm{C}$ approximating a single Debye peak. The peak of the relaxation occurs at $3 \mathrm{kHz}$; nearly identical in frequency and temperature to the electrical relaxation noted in Figs. 6 and 7. As the amount of yttria increases the internal friction peak also increases. Based upon the magnitude, temperature and frequency of this peak, point defects are the likely mechanism. Hence by controlling the concentration of yttria we are able to tailor the population of defect clusters which may influence the domain mechanics of this material. Both the anelastic and elastic temperature spectrum provides additional evidence of minimal residual glassy phases at the grain boundaries. 
Summary of Findings

- Additions of yttria do not degrade the electromechanical properties of lanthanum titanate.

- $\mathrm{Y}_{2} \mathrm{O}_{3} / \mathrm{La}_{2} \mathrm{Ti}_{2} \mathrm{O}_{7}$ exhibits extremely low internal friction and hence has mechanical fatigue resistant properties at low strains. .

- Micromechanical observations manifested themselves in the electrical nature of these materials.

- Both the electrical and mechanical relaxations appear to be thermally activated processes.

References:

1.Scheunemann K. and Muller-Bushbaum, H.K. "Zur Kristallstruktur von La2Ti2O7” Journal of Inorganic Nuclear Chemistry, volume 37, 1975 pp.1879-1881.

2. Kimura M, Matsushi.S, Nanamats.S, Takahash.M, Doi K

Electro optic and piezoelectric properties of La2Ti2O7 Single-Crystal, Japanese Journal Of Applied Physics volume. 11 number 6: 9041972.

3. Cox, P.A. The Electronic Structure and Chemistry of Solids, Oxford University Press, 1987. 


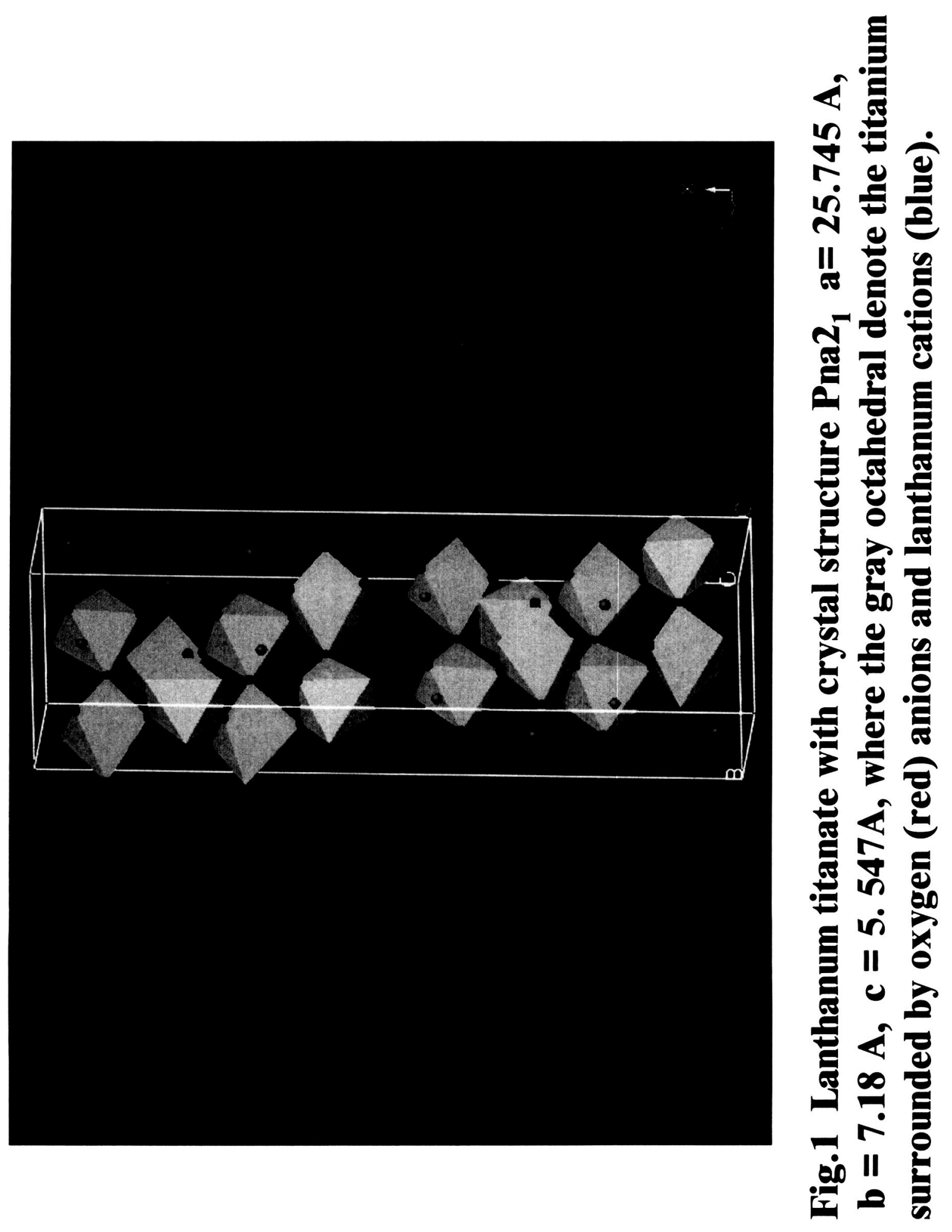




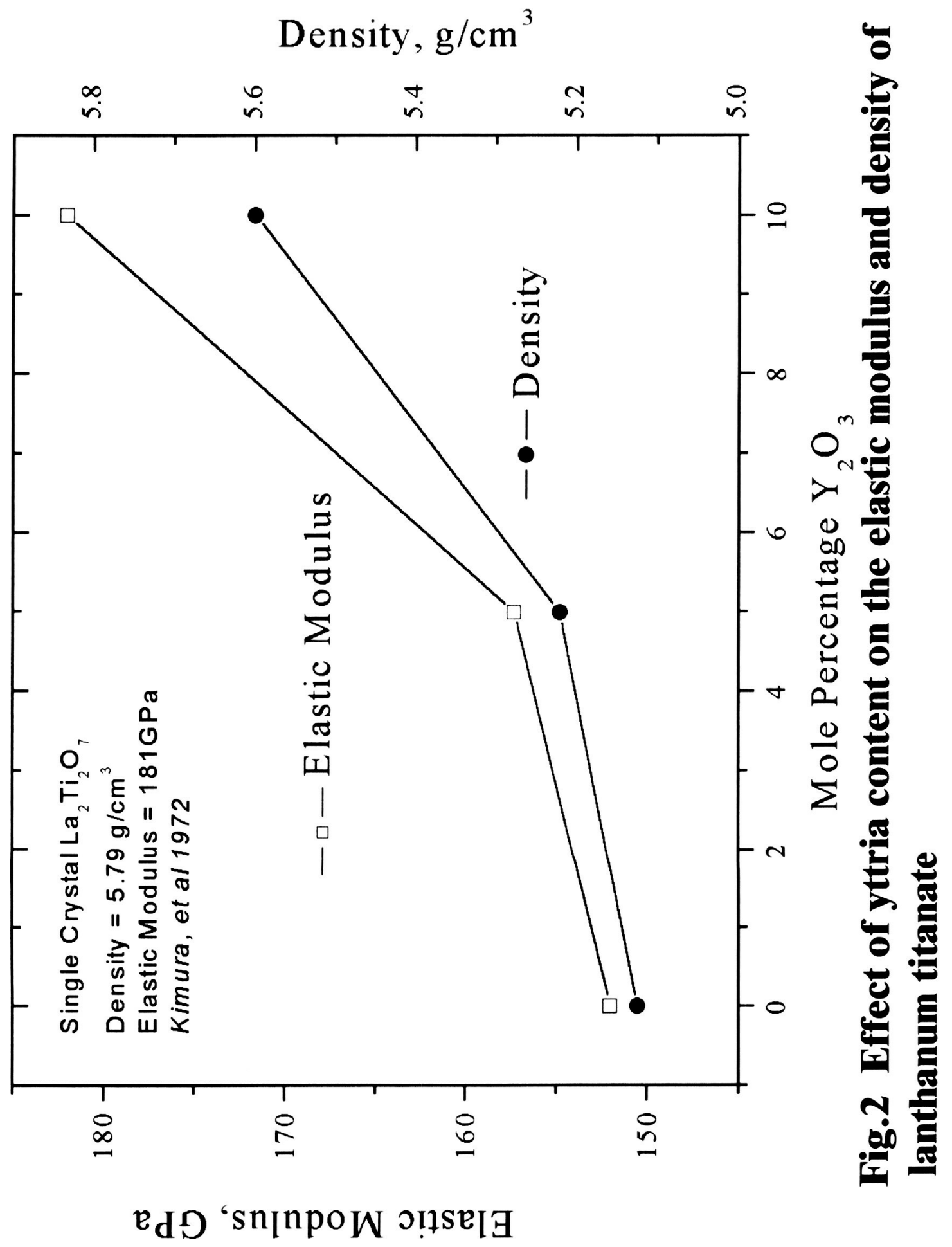




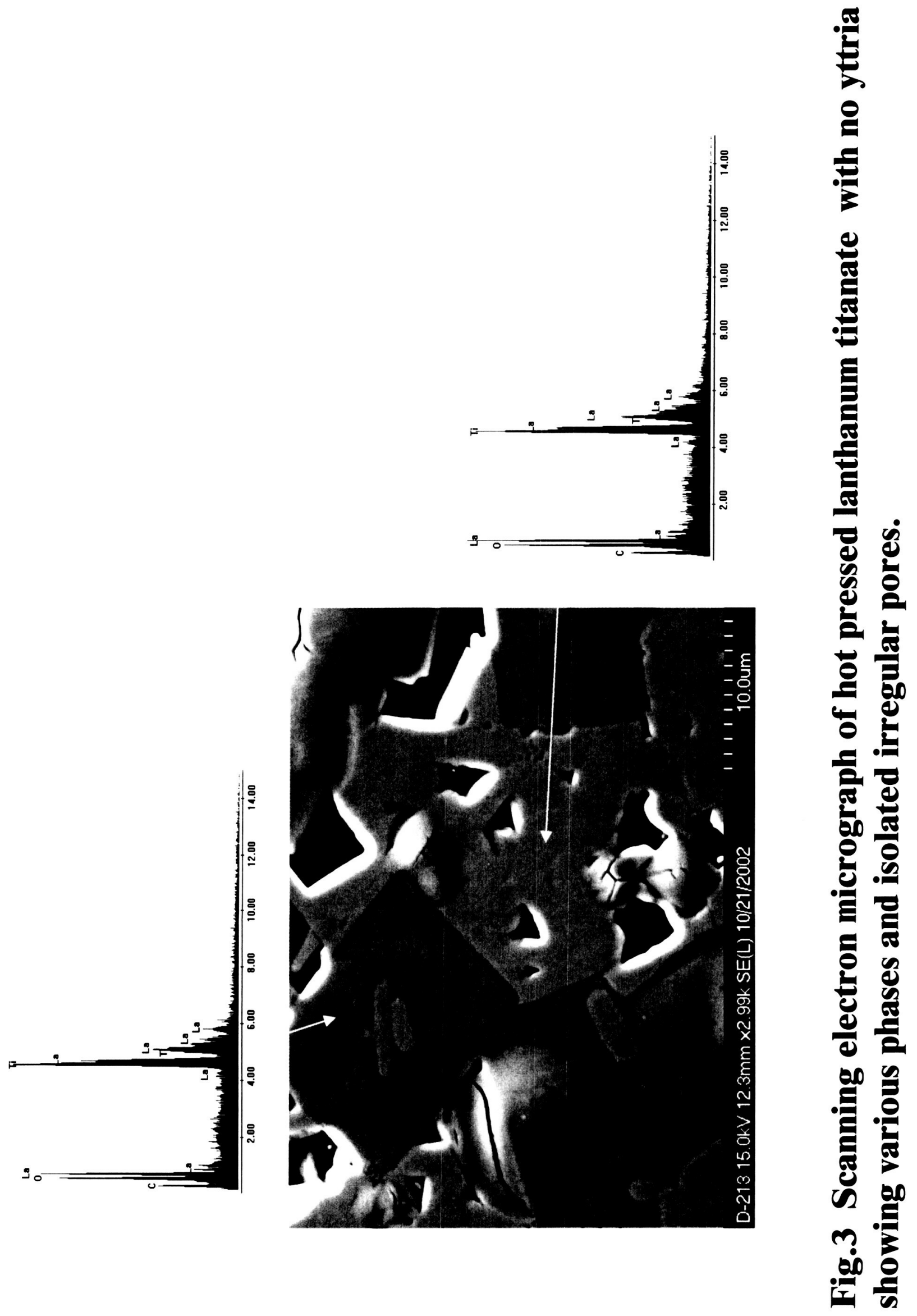




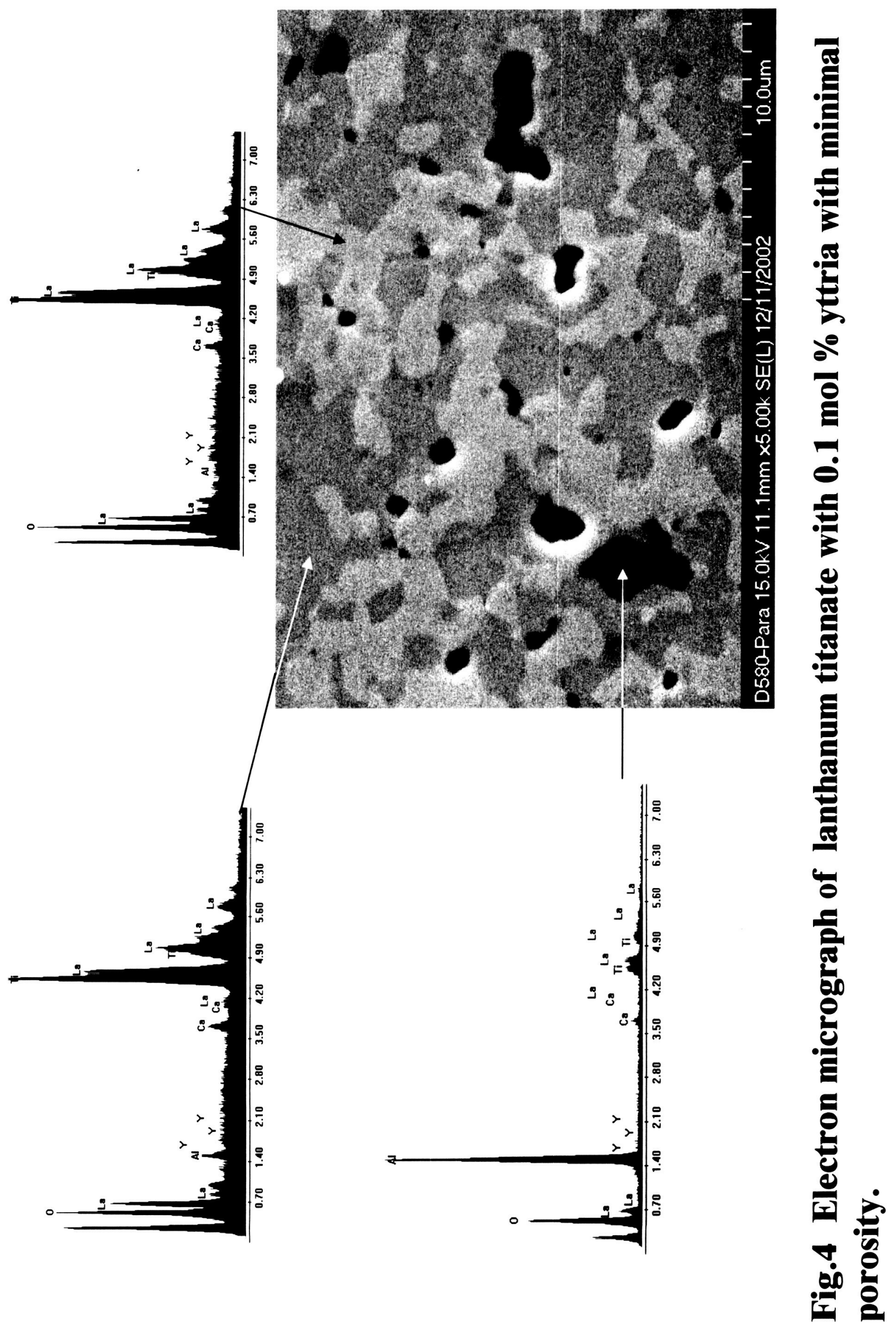




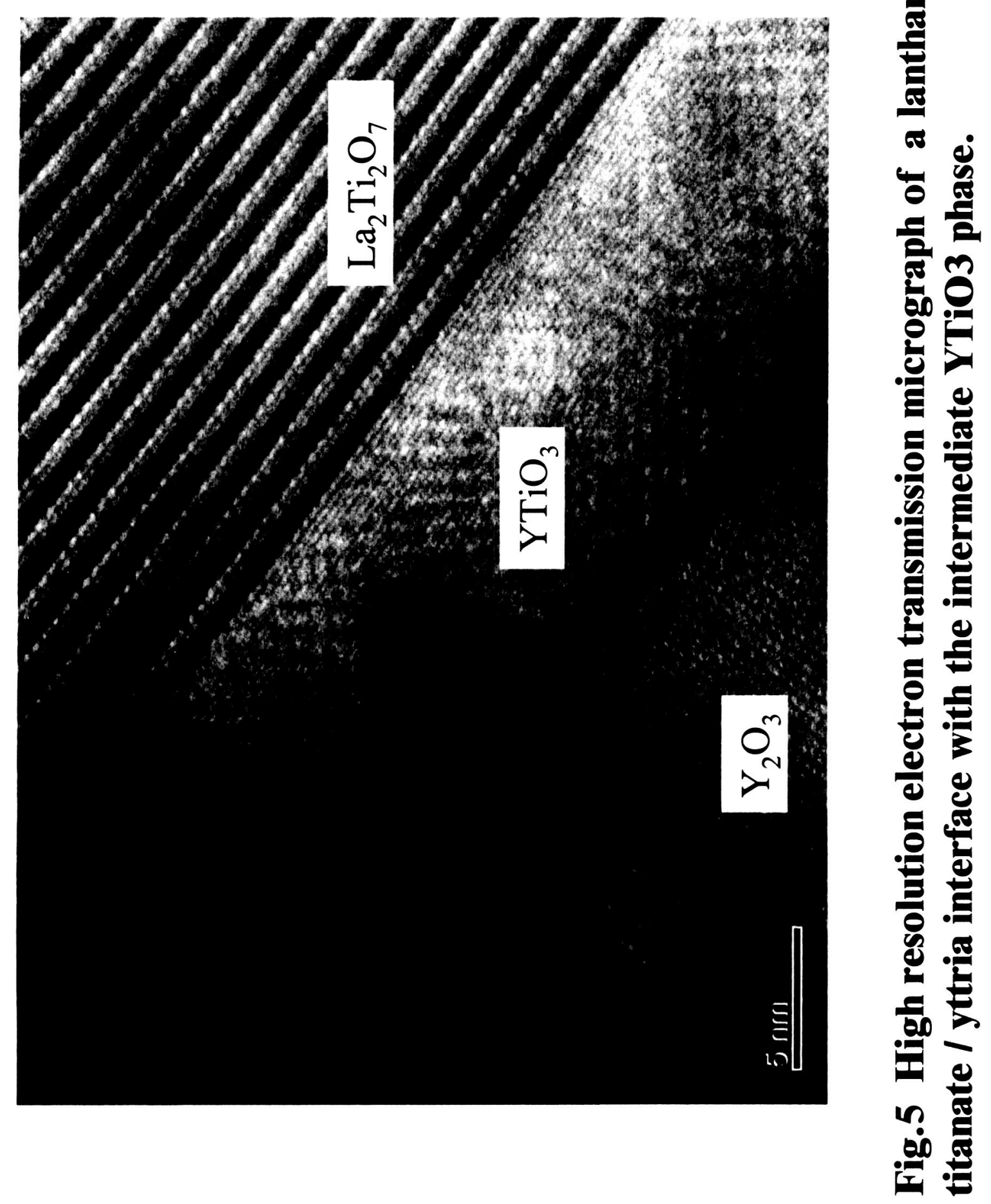




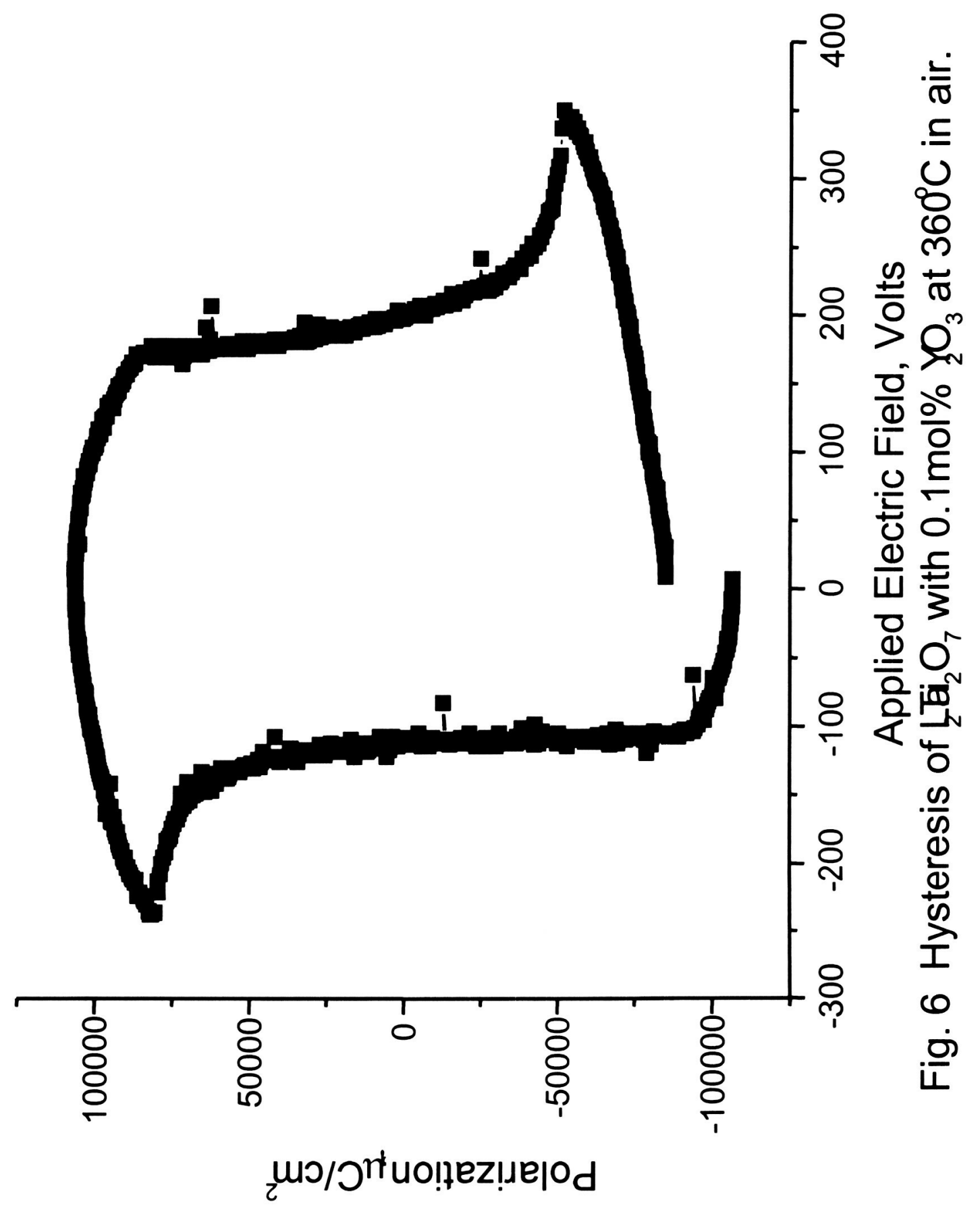




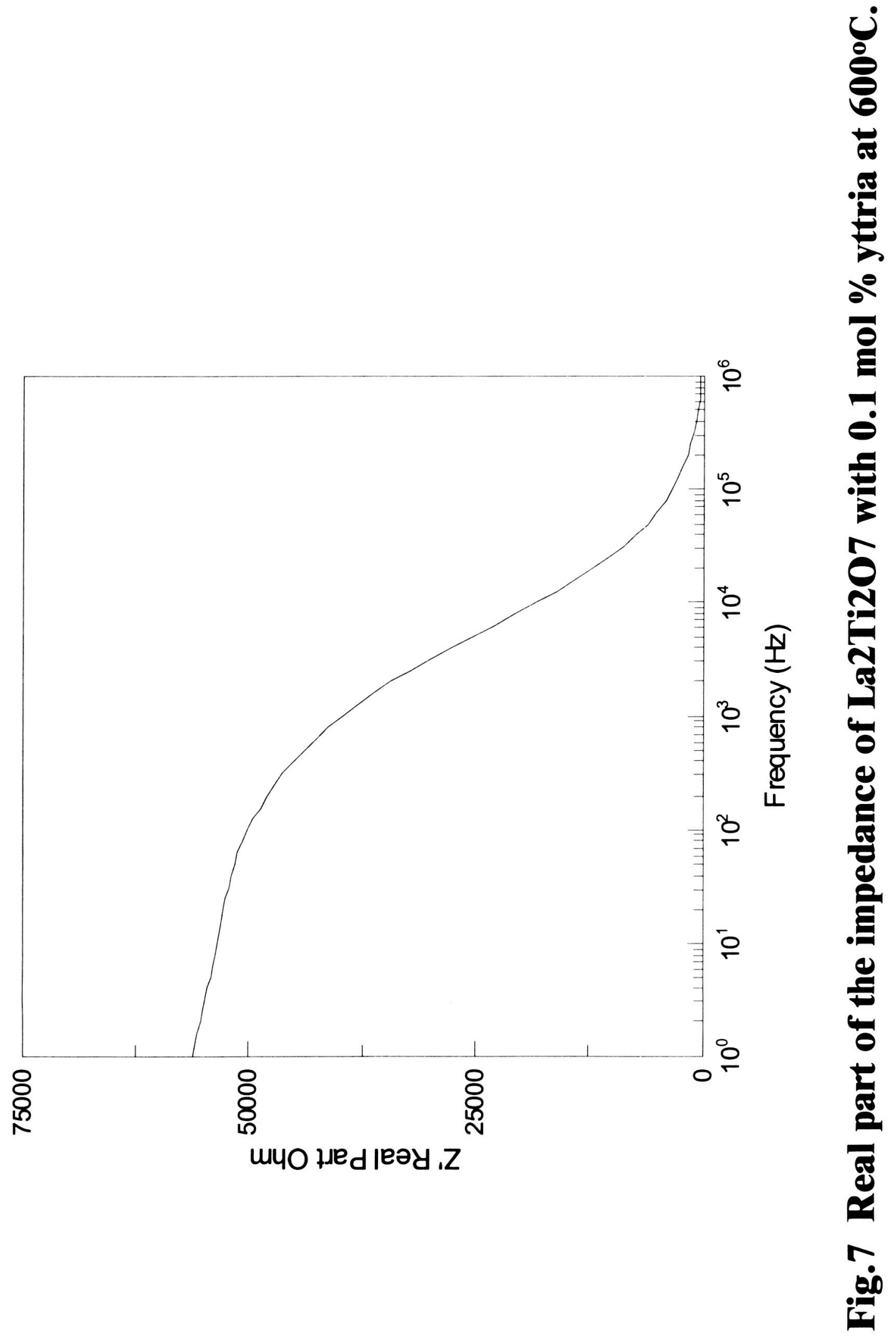




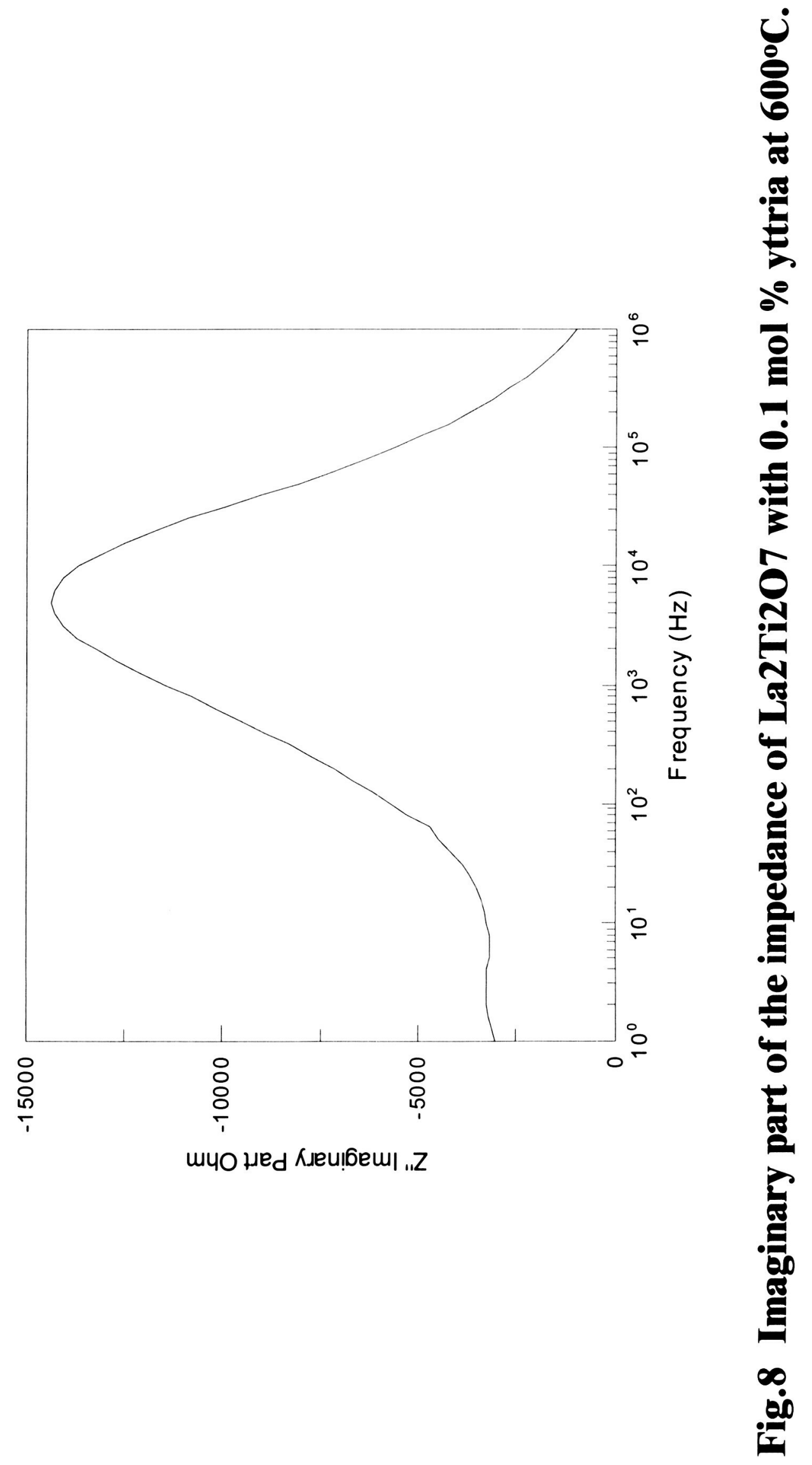




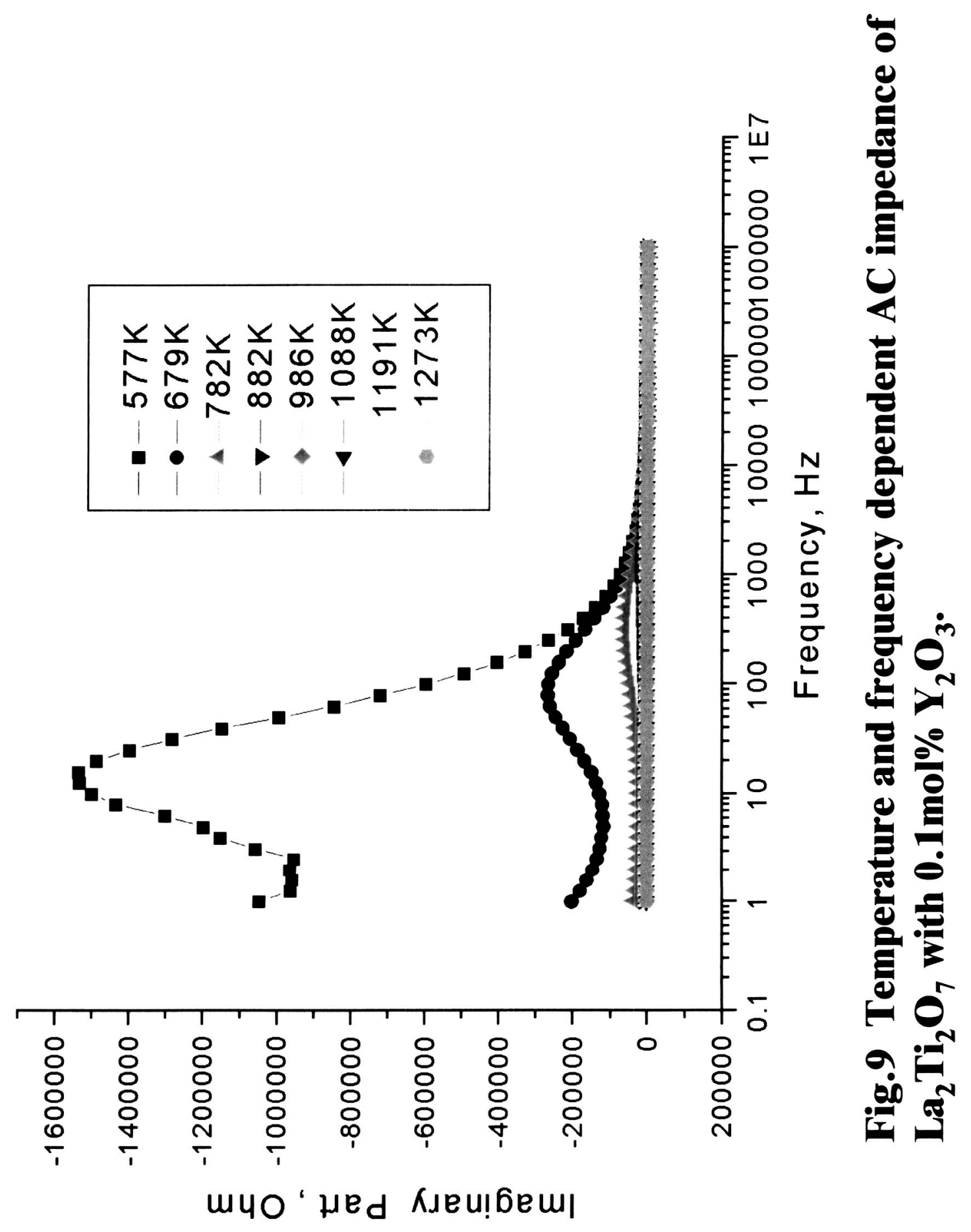




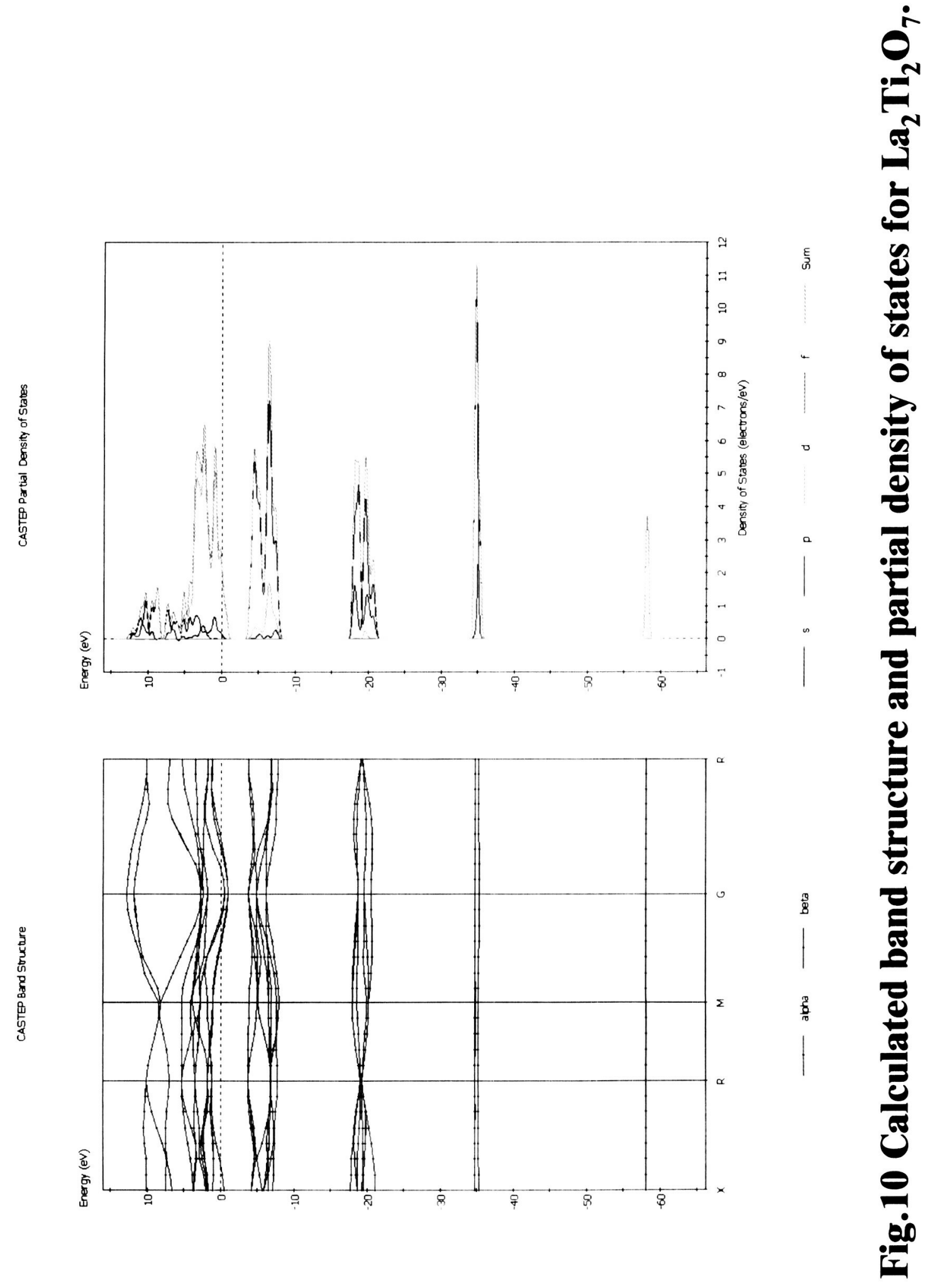




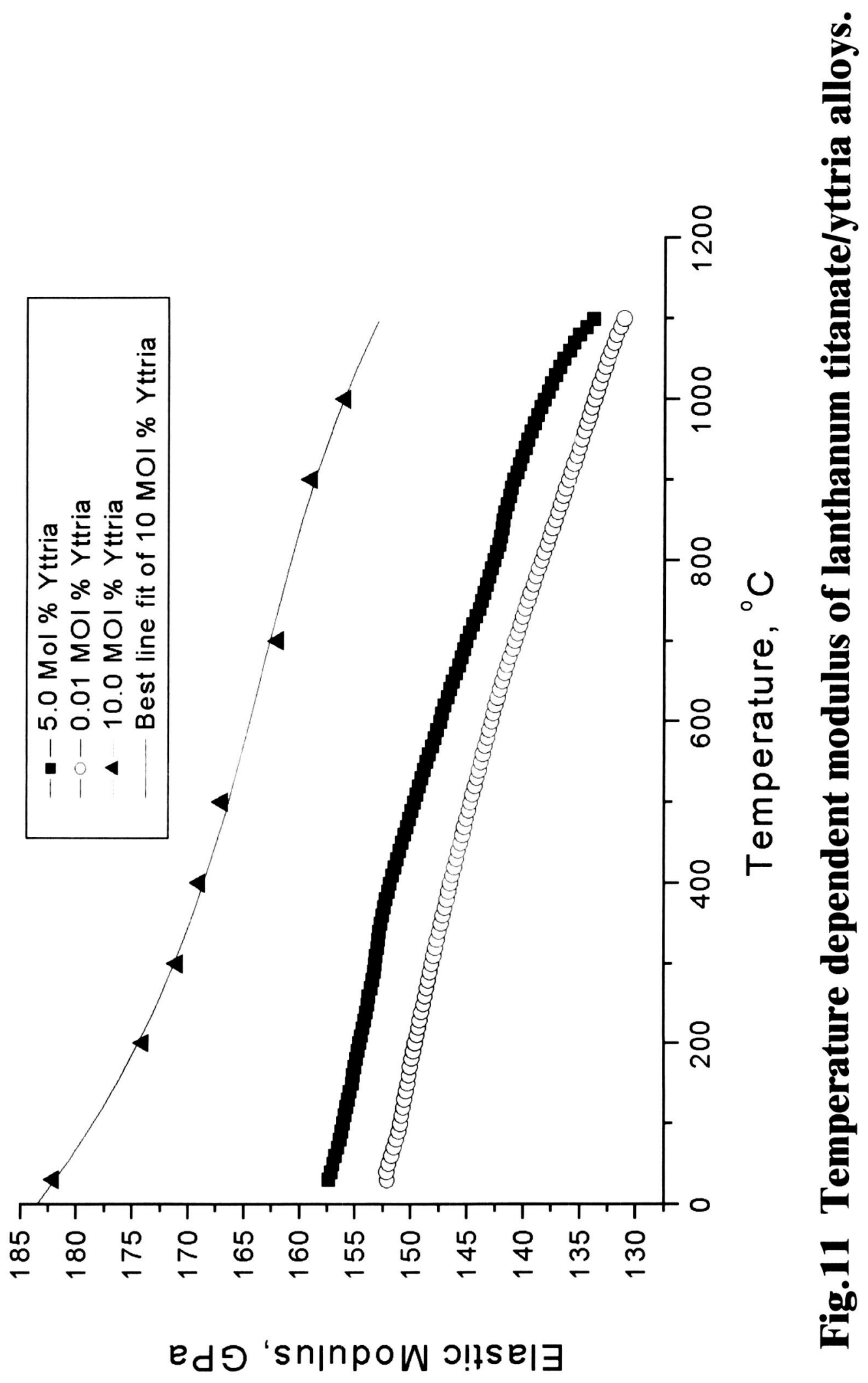




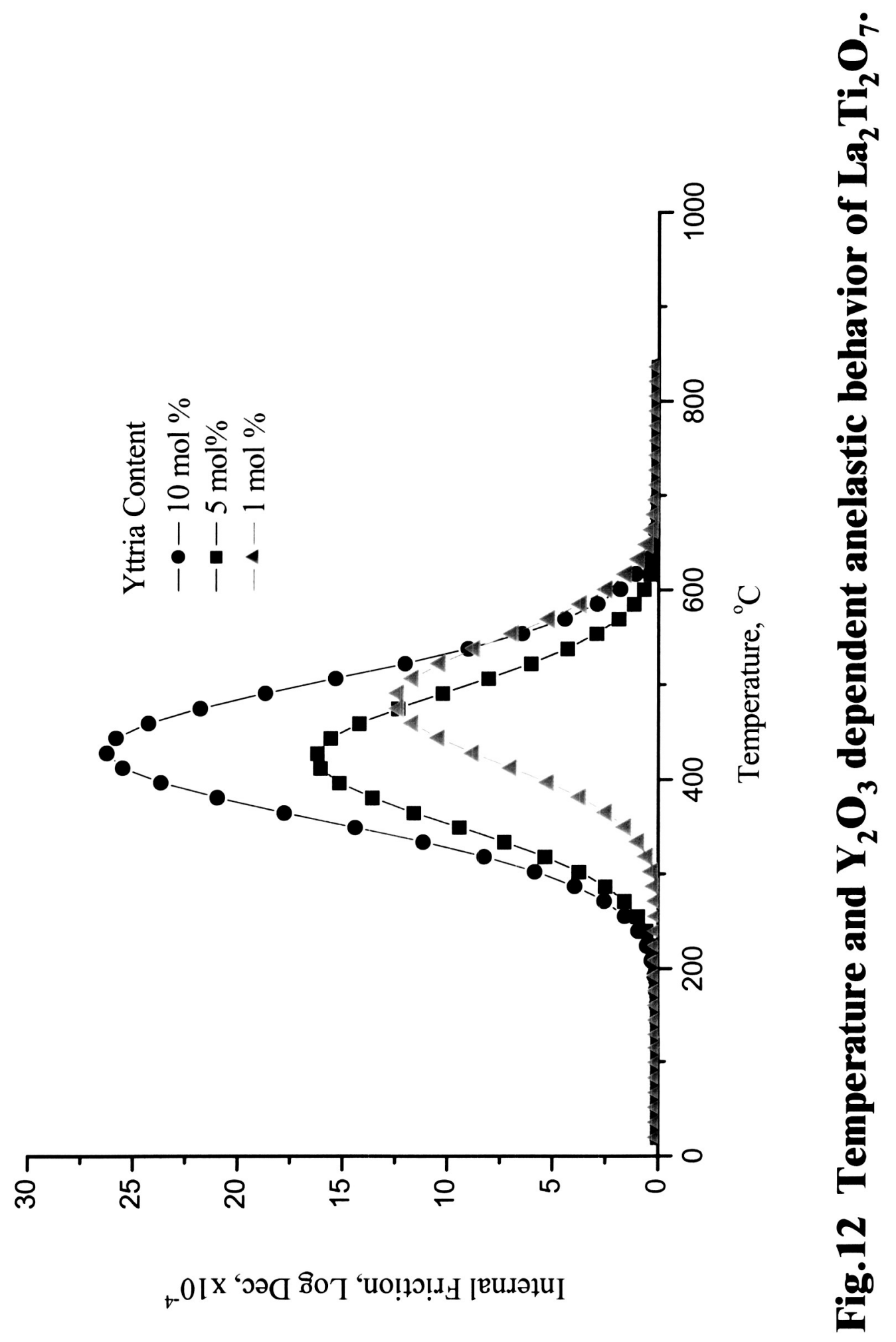

